\title{
Metabolic syndrome and serum carotenoids: findings of a cross-sectional study in Queensland, Australia
}

\author{
Terry Coyne $^{1 *}$, Torukiri I. Ibiebele ${ }^{2}$, Peter D. Baade ${ }^{3,4}$, Christine S. McClintock ${ }^{5}$ and Jonathan E. Shaw ${ }^{6}$ \\ ${ }^{1}$ School of Population Health, University of Queensland, Brisbane, QLD, Australia \\ ${ }^{2}$ Queensland Institute of Medical Research, Brisbane, QLD, Australia \\ ${ }^{3}$ Viertel Center for Research in Cancer Control, The Cancer Council Queensland, Brisbane, QLD, Australia \\ ${ }^{4}$ School of Public Health, Queensland University of Technology, Brisbane, QLD, Australia \\ ${ }^{5}$ Center for Military and Veterans' Health, University of Queensland, Brisbane, QLD, Australia \\ ${ }^{6}$ Baker IDI Heart and Diabetes Institute, Melbourne, Vic., Australia
}

(Received 12 September 2008 - Revised 1 April 2009 - Accepted 26 May 2009 - First published online 27 July 2009)

Several components of the metabolic syndrome, particularly diabetes and CVD, are known to be oxidative stress-related conditions and there is research to suggest that antioxidant nutrients may play a protective role in these conditions. Carotenoids are compounds derived primarily from plants and several have been shown to be potent antioxidant nutrients. The aim of the present study was to examine the associations between metabolic syndrome status and major serum carotenoids in adult Australians. Data on the presence of the metabolic syndrome, based on International Diabetes Federation 2005 criteria, were collected from 1523 adults aged 25 years and over in six randomly selected urban centres in Queensland, Australia, using a cross-sectional study design. Weight, height, BMI, waist circumference, blood pressure, fasting and $2 \mathrm{~h}$ blood glucose and lipids were determined, as well as five serum carotenoids. Mean serum $\alpha$-, $\beta$-carotenes and the sum of the five carotenoid concentrations were significantly lower $(P<0.05)$ in persons with the metabolic syndrome (after adjusting for age, sex, education, BMI status, alcohol intake, smoking, physical activity status and vitamin/mineral use) than persons without the syndrome. $\alpha-, \beta$ - and total carotenoids also decreased significantly $(P<0.05)$ with increased number of components of the metabolic syndrome, after adjusting for these confounders. These differences were significant among former smokers and non-smokers, but not in present smokers. Low concentrations of serum $\alpha$-, $\beta$-carotenes and the sum of five carotenoids appear to be associated with metabolic syndrome status. Additional research, particularly longitudinal studies, may help to determine whether these associations are causally related to the metabolic syndrome, or are a result of the pathologies of the syndrome.

Carotenoids: Metabolic syndrome: Cross-sectional studies

Although there is no universal definition of the metabolic syndrome, it is generally described as a constellation of pathologies or anthropometric conditions, which include central obesity, glucose intolerance, lipid abnormalities and hypertension. It is, however, universally accepted that the presence of the metabolic syndrome is associated with increased risk of type 2 diabetes and $\mathrm{CVD}^{(1,2)}$.

The prevalence of the metabolic syndrome in developed countries varies widely depending upon definitions used and age ranges included, but is estimated to be $24 \%$ among adults 20 years and over in the USA ${ }^{(3)}$. Given the impending worldwide epidemic of obesity, diabetes and CVD, strategies aimed at greater understanding of the pathology of the syndrome as well as strategies aimed at preventing or treating persons with the syndrome are urgently required.

Both diabetes and CVD are known to be oxidative stressrelated conditions and some researchers ${ }^{(4)}$ suggest that antioxidant nutrients may play a protective role in these conditions. Several cross-sectional surveys ${ }^{(5-7)}$ have found lower levels of serum carotenoids among those with impaired glucose metabolism or type 2 diabetes. Carotenoids are compounds derived primarily from plants and several have been shown to be potent antioxidants ${ }^{(8)}$. There is considerable epidemiological evidence that some serum carotenoids may play a protective role against the development of chronic diseases such as atherosclerosis ${ }^{(9,10)}$, stroke $^{(11)}$, hypertension $^{(12)}$, certain cancers ${ }^{(10,13)}$, inflammatory diseases ${ }^{(14)}$ and diabetic retinopathy ${ }^{(15)}$. The primary carotenoids found in human serum are $\alpha$-, $\beta$-carotenes, $\beta$-cryptoxanthin, lutein/ zeaxanthin and lycopene ${ }^{(16)}$.

Several studies have found an association between fruit and vegetable intakes, or dietary patterns rich in fruit and vegetables, with a lower risk of the metabolic syndrome ${ }^{(17-20)}$. Other studies, however, have failed to find such associations ${ }^{(21)}$. Results of clinical trials testing supplements of these nutrients with chronic diseases, however, have not been promising ${ }^{(22)}$.

Few studies have investigated associations of antioxidant nutrients and the metabolic syndrome. Ford et al. ${ }^{(23)}$

Abbreviation: NHANES, National Health and Nutrition Examination Survey. 
reported lower levels of several carotenoids and vitamins $\mathrm{C}$ and $\mathrm{E}$ among those with metabolic syndrome present compared with those without the syndrome in the Third National Health and Nutrition Examination Survey (NHANES III). Sugiura et al. ${ }^{(24)}$ suggested that several carotenoids may exert a protective effect against the development of the metabolic syndrome, especially among present smokers. Confirming these findings in another population may add strength to these associations.

In the present study, we investigated the relationships between these five primary serum carotenoids and the metabolic syndrome in a cross-sectional population-based study in Queensland, Australia.

\section{Subjects and methods}

\section{Subjects}

The study was conducted in Queensland, Australia, between October and December 2000 as part of a national study, the Australian Diabetes, Obesity and Lifestyle Study, to determine the prevalence of diabetes and associated cardiovascular risk factors among adults aged 25 years and over. Details of the sampling framework, overall study design, methods and central findings have been published elsewhere ${ }^{(25,26)}$. The study was approved by the International Diabetes Institute and the University of Queensland ethics committees. Six urban sites were randomly selected from census collector districts in Queensland. The collector districts were selected without replacement and with probability proportional to size. Non-institutionalised adults aged 25 years and over residing in private dwellings were included in the survey if they had resided permanently at the address for a minimum of 6 months before the survey. Persons with physical or intellectual disabilities that precluded participation in the study were not included.

Trained interviewers conducted house-to-house interviews and eligible participants were invited to attend a biomedical examination, which included the collection of blood samples, blood pressure determinations, anthropometric measurements and the administration of standardised questionnaires related to diet as well as socio-demographic, lifestyle and healthrelated characteristics. All respondents gave informed consent to participate in the survey upon arrival at the testing site. A total of 1536 persons (890 females and 646 males) in Queensland completed the physical examination. Although the overall response rate in the study was low $(70 \%$ of eligible adults took part in the initial household survey and of those $55 \%$ completed the physical examination), the internal validity and quality control of the data collection were of high quality ${ }^{(26)}$.

\section{Methods}

Study participants arrived for the examination having fasted for at least $12 \mathrm{~h}$. Blood pressure measurements were taken using Dinamap sphygmomanometer. Blood was drawn for fasting glucose. Participants not taking hypoglycaemic medication completed an oral glucose tolerance test $(2 \mathrm{~h}$ after consuming a $75 \mathrm{~g}$ glucose drink). Fasting and 2-h $(2 \mathrm{~h})$ plasma glucose were measured enzymatically (glucose oxidase) on an Olympus AU600 analyser. Participants were also classified as having diabetes if they were receiving treatment for diabetes in the form of tablets or insulin at the time of the study.

Lipids, total cholesterol, HDL cholesterol (HDL) and TAG were measured enzymatically on an Olympus AU 600. LDL cholesterol was calculated from the equation of Friedewald et al. ${ }^{(27)}$ :

$$
\mathrm{LDL}=\text { total cholesterol }-(\mathrm{HDL}+(\mathrm{TAG} / 5)) .
$$

Blood was drawn for the carotenoid determinations at the time of the $2 \mathrm{~h}$ oral glucose tolerance test, or $2 \mathrm{~h}$ after the fasting sample for those who did not have the oral glucose tolerance test. Serum samples were meticulously handled and protected from light at each stage of processing to prevent deterioration and degradation. The serum was pipetted, frozen, packed in dry ice and shipped to the laboratory in Brisbane and were analysed within 3 weeks of collection. The five serum carotenoids were assayed simultaneously according to the HPLC procedure described by Talwar et al. ${ }^{(28)}$. The intra-batch $\mathrm{CV}$ by this method were $6 \cdot 5,7 \cdot 6$, $7.3,6.9$ and $9.0 \%$ for $\alpha-\beta$-carotenes, $\beta$-cryptoxanthin, lutein and lycopene, respectively, and the inter-batch (analysed after stored at $-70^{\circ} \mathrm{C}$ over a period of 8 weeks) $\mathrm{CV}$ were $13,9.6,8 \cdot 7,8.5$ and $11 \%$, respectively.

The diagnostic criteria for the metabolic syndrome are based on the 2005 International Diabetes Federation definition ${ }^{(29)}$. According to the International Diabetes Federation, to be identified as having the metabolic syndrome, a person must have central obesity (defined as waist circumference $\geq 94 \mathrm{~cm}$ for Europid men and $\geq 80 \mathrm{~cm}$ for Europid women, with ethnicity specific values for other groups) plus any two of the following four factors: raised serum TAG level $(\geq 1.7 \mathrm{mmol} / \mathrm{l})$; reduced serum HDL cholesterol $(<1.03 \mathrm{mmol} / \mathrm{l}$ in males and $<1.29 \mathrm{mmol} / \mathrm{l}$ in females - or specific treatment for these lipid abnormalities); raised blood pressure (systolic blood pressure $\geq 130 \mathrm{mmHg}$ or diastolic $\geq 85 \mathrm{mmHg}$ - or treatment of previously diagnosed hypertension); impaired fasting glycaemia (fasting plasma glucose $\geq 5.6 \mathrm{mmol} / \mathrm{l}$ or previous diagnosed type 2 diabetes).

Of those diagnosed with diabetes, $2.5 \%$ were classified as having type 1 diabetes and were excluded from the analysis because they do not share common aetiological factors such as obesity, increasing age, nutrition or physical activity with metabolic syndrome. Participants were defined as having type 1 diabetes if insulin treatment had been started within 2 years of diagnosis, and, if they were 40 years of age or older when diagnosed, their current BMI was $<27$.

Demographic and lifestyle variables were collected using standardised questionnaires. Height and weight were measured by trained personnel and BMI was calculated as weight (in $\mathrm{kg}$ )/height (in $\mathrm{m}^{2}$ ). BMI was categorised as: obese $(\mathrm{BMI} \geq 30$ ); overweight $(\mathrm{BMI} \geq 25$ to $<30$ ); normal weight $(\mathrm{BMI}<25)$. As there were only eighteen participants classified as underweight (BMI $<18.5$ ), they were grouped with the normal weight group.

Waist circumference was measured halfway between the lower border of the ribs and the iliac crest on a horizontal plane. Using a steel-measuring tape, two measurements were recorded. If the measurements varied by more than $2 \mathrm{~cm}$, 
a third measurement was taken. The mean of the two closest measurements was calculated.

Blood pressure measurement was performed in a seated position after participants had rested for at least $5 \mathrm{~min}$, using a Dinamap semi-automatic oscillometric recorder. An appropriate cuff size was used and the arm was supported by a table at heart level. Three readings were taken at 1 min intervals. The mean of the first two readings was recorded. If the difference between the three readings was $>10 \mathrm{mmHg}$, the mean of the two closest measurements was used.

Physical activity beneficial to health was categorised as: sufficiently active ( $>150 \mathrm{~min}$ 'physical activity time' in the previous week); insufficiently active but not sedentary ( $<150$ min physical activity time in the previous week); sedentary (no participation in physical activity in the previous week). Physical activity time was calculated as the sum of the time spent walking, or performing moderate activity plus double the time spent in vigorous activity to reflect its greater intensity.

Vitamin supplement use during the previous $24 \mathrm{~h}$ was categorised as yes for respondents who indicated that they took any vitamin or mineral supplements on the previous day, and no for respondents who indicated they did not.

Criteria used to assess the number of metabolic syndrome components present in a participant using the 2005 International Diabetes Federation ${ }^{(29)}$ definition are as follows: components 0 - none of the metabolic syndrome components (i.e. abdominal obesity, raised TAG, reduced HDL cholesterol, raised blood pressure and impaired fasting plasma glucose) are present; components 1 - any one of the five metabolic syndrome components is present; components 2 - any two of the five components are present; components 3 - any three of the components are present; components 4 any four of the components are present; components 5 - all five metabolic syndrome components are present.

Participants were asked, 'How many serves of vegetables do you usually eat each day? Including fresh, frozen or tinned vegetables (A serve $=$ half cup cooked vegetables or one cup of salad vegetables)'. Usual consumption of fruit was assessed by the question, 'How many serves of fruit do you usually eat each day? Including fresh, frozen or tinned fruit (A serve $=$ one medium piece or two small pieces of fruit or one cup of diced pieces of fruit)'. Participants were categorised into three groups according to their responses to both questions; $\leq 1$ serve, $2-3$ serves and $\geq 4$ serves.

\section{Statistical analyses}

Data were analysed using the survey (svy) commands in STATA statistical software version 8 (Stata Corporation, College Station, TX, USA) ${ }^{(30)}$. These commands take into account the complex cluster survey design in the calculation of estimates, variance, standard errors and CI. Age-adjusted means or percentages for selected baseline characteristics were weighted for age and sex to the Queensland population aged over 25 years for the survey year.

Pearson's $\chi^{2}$-statistic was used to assess the relationship between the presence and absence of the metabolic syndrome and selected categorical variables. Student's $t$ test was used to compare differences in means between two groups; ANOVA was used to assess overall differences in means between serum carotenoids and variables with more than two groups.

Distributions of serum carotenoids were skewed and therefore natural logarithmically transformed to better approximate the normal distribution for regression analyses. Association between log-transformed serum carotenoids as dependent variables and metabolic syndrome status were assessed using multiple linear regression analysis. Results are reported as back-transformed geometric means. Analysis was performed for each serum carotenoid separately, and the sum of the five carotenoids, adjusting for the following potential confounders: age; sex; education; BMI; smoking; alcohol intake; physical activity; vitamin use. We further performed stratified analysis by smoking status to further investigate whether the association between serum carotenoids and metabolic syndrome is modified by smoking status.

The confounders were included simultaneously into the model. Due to missing values, the sample size is not the same for all analyses.

\section{Results}

The prevalence of the metabolic syndrome by demographic and health-related characteristics is provided in Table 1 . The overall prevalence of the syndrome was $24 \%$ and was significantly higher among males than females. As would be expected, significant differences in prevalence of the syndrome were seen with BMI, waist circumference, systolic and diastolic blood pressure and blood lipids. Significant differences were also evident by age group, smoking status, educational status and income. Income was marginally inversely associated. The prevalence increased with age, and was lower in those with post-graduate education. No significant differences were seen by alcohol intake, physical activity levels, vitamin usage or fruit intake. There was actually an inverse relationship between vegetable intake (not fruit) and serum carotenoids. Those who consumed four serves or more of vegetable were less likely to have the metabolic syndrome compared with those who consumed one serve or less of vegetables.

Table 2 provides the adjusted geometric mean of each of the serum carotenoids by metabolic syndrome status. The mean concentrations of serum $\alpha-, \beta$-carotenes and the sum of the five carotenoids were significantly lower for participants with the metabolic syndrome present compared with those without the syndrome, after adjusting for potential confounding variables including age, sex, educational status, BMI, smoking, alcohol intake, physical activity and vitamin use.

Table 3 shows that concentrations of $\alpha-, \beta$-carotenes and the sum of the five carotenoids decreased significantly, as the number of components of the metabolic syndrome increased after adjusting for potential confounding variables as above. Table 3 also indicates the percentage of participants with none, one, two, three, four or all five of the components.

Similarly, there was an inverse association between quartiles of individual and total serum carotenoids and metabolic syndrome status and each of its components as shown in Table 4. The strength of these associations was strongest for $\alpha-, \beta$-carotenes and the sum of the five carotenoids.

Table 5 looks at the effect of smoking status on carotenoid concentrations and metabolic syndrome. No significant 
Table 1. Age-adjusted means or percentages for selected baseline characteristics of the Australian Diabetes, Obesity and Lifestyle Study participants aged 25 years and over by metabolic syndrome status

(Percentages with their standard error values; $n 1523^{*}$ )

\begin{tabular}{|c|c|c|c|c|c|c|c|}
\hline \multirow[b]{3}{*}{ Selected characteristics (\%, mean) } & \multicolumn{7}{|c|}{ Metabolic syndrome status } \\
\hline & \multicolumn{2}{|c|}{ Present } & & \multicolumn{2}{|c|}{ Absent } & & \multirow[b]{2}{*}{$P \ddagger$} \\
\hline & $n$ & Percentage $\dagger$ & & $n$ & Percentage $†$ & & \\
\hline \multicolumn{7}{|l|}{$\operatorname{Sex}(\%) \S$} & 0.05 \\
\hline Male & 195 & $26 \cdot 7$ & & 451 & $73 \cdot 3$ & & \\
\hline Female & 229 & $21 \cdot 3$ & & 661 & 78.7 & & \\
\hline All persons & 424 & $24 \cdot 0$ & & 1112 & $76 \cdot 0$ & & \\
\hline \multicolumn{7}{|l|}{ Age group (years, \%)§ } & $<0.01$ \\
\hline $25-34$ & 19 & 11.0 & & 161 & 89.0 & & \\
\hline $35-44$ & 48 & $14 \cdot 6$ & & 275 & 85.4 & & \\
\hline $45-54$ & 114 & 29.4 & & 270 & $70 \cdot 6$ & & \\
\hline $55-64$ & 120 & 38.4 & & 199 & $61 \cdot 7$ & & \\
\hline $65-74$ & 88 & 38.0 & & 145 & $62 \cdot 0$ & & \\
\hline $75+$ & 35 & $36 \cdot 2$ & & 62 & 63.8 & & \\
\hline \multicolumn{7}{|l|}{ Educational status (\%) } & 0.04 \\
\hline Secondary school or less & 191 & $27 \cdot 5$ & & 415 & $72 \cdot 5$ & & \\
\hline Trade certificate, bachelor's degree & 214 & $23 \cdot 0$ & & 598 & $77 \cdot 0$ & & \\
\hline Post-graduate qualification & 19 & $15 \cdot 8$ & & 95 & 84.2 & & \\
\hline \multicolumn{7}{|l|}{ Income (\%) } & 0.06 \\
\hline$\$ 800-1500+/$ week & 136 & 19 & & 465 & 81 & & \\
\hline$\$ 400-799 /$ week & 133 & 24 & & 327 & 76 & & \\
\hline \$0-399/week & 147 & 30 & & 299 & 70 & & \\
\hline \multicolumn{7}{|l|}{ BMI (\%) } & $<0.01$ \\
\hline Obese $(\mathrm{BMI}>30)$ & 208 & $57 \cdot 2$ & & 149 & $42 \cdot 8$ & & \\
\hline Overweight (BMI $\geq 25$ to $<30$ ) & 189 & $28 \cdot 3$ & & 379 & $71 \cdot 7$ & & \\
\hline Normal $(\mathrm{BMI}<25)$ & 27 & $3 \cdot 2$ & & 584 & $96 \cdot 8$ & & \\
\hline \multicolumn{7}{|l|}{ Smoking status (\%) } & 0.05 \\
\hline Current & 59 & $25 \cdot 1$ & & 147 & 74.9 & & \\
\hline Former smokers & 142 & $29 \cdot 8$ & & 289 & $70 \cdot 2$ & & \\
\hline Never smoked & 218 & $20 \cdot 8$ & & 660 & $79 \cdot 2$ & & \\
\hline \multicolumn{7}{|l|}{ Alcohol intake (\%) } & 0.12 \\
\hline None & 105 & $28 \cdot 0$ & & 233 & $72 \cdot 0$ & & \\
\hline$\leq 60$ standard drinks/month & 265 & $22 \cdot 1$ & & 761 & 77.9 & & \\
\hline$>60$ standard drinks/month & 54 & $27 \cdot 8$ & & 118 & $72 \cdot 2$ & & \\
\hline \multicolumn{7}{|l|}{ Physical activity beneficial to health (\%) } & 0.07 \\
\hline Sufficiently active & 179 & $22 \cdot 0$ & & 542 & 78.0 & & \\
\hline Insufficiently active & 134 & $22 \cdot 2$ & & 380 & $77 \cdot 8$ & & \\
\hline Sedentary & 111 & 33.1 & & 185 & $66 \cdot 9$ & & \\
\hline \multicolumn{7}{|l|}{ Vitamin use (\%) } & 0.81 \\
\hline No & 265 & 23.4 & & 705 & $76 \cdot 6$ & & \\
\hline Yes & 116 & $22 \cdot 8$ & & 340 & $77 \cdot 2$ & & \\
\hline \multicolumn{7}{|l|}{ Fruit intake (serves/d) \% } & 0.68 \\
\hline$\leq 1$ Serve & 72 & $25 \cdot 6$ & & 177 & 74.4 & & \\
\hline $1-3$ Serves & 219 & 23.4 & & 586 & $76 \cdot 6$ & & \\
\hline$\geq 4$ Serves & 123 & 24.5 & & 329 & 75.5 & & \\
\hline Vegetable intake (serves/d) \% & & & & & & & 0.02 \\
\hline$\leq 1$ Serve & 168 & $25 \cdot 0$ & & 426 & $75 \cdot 0$ & & \\
\hline $1-3$ Serves & 204 & $24 \cdot 3$ & & 522 & $75 \cdot 7$ & & \\
\hline$\geq 4$ Serves & 41 & $20 \cdot 1$ & & 141 & 79.9 & & \\
\hline $\mathrm{BP}(\mathrm{mmHg})$ & & Mean & SE & & Mean & SE & \\
\hline Systolic BP & 420 & $137 \cdot 4$ & 1.9 & 1104 & $121 \cdot 6$ & $1 \cdot 2$ & $<0.01$ \\
\hline Diastolic BP & 421 & 73.4 & 1.2 & 1105 & 65.8 & 1.1 & $<0.01$ \\
\hline Blood lipids (mmol/l) & & & & & & & \\
\hline HDL cholesterol & 424 & $1 \cdot 15$ & 0.03 & 1112 & 1.46 & 0.01 & $<0.01$ \\
\hline TAG & 424 & $2 \cdot 54$ & 0.14 & 1112 & $1 \cdot 13$ & 0.03 & $<0.01$ \\
\hline LDL cholesterol & 377 & $3 \cdot 70$ & 0.07 & 1106 & $3 \cdot 37$ & 0.04 & 0.02 \\
\hline Fasting plasma glucose $(\mathrm{mmol} / \mathrm{l})$ & 424 & $5 \cdot 74$ & 0.08 & 1112 & $5 \cdot 10$ & 0.02 & $<0.01$ \\
\hline Waist circumference $(\mathrm{cm})$ & & & & & & & \\
\hline Males & 195 & $106 \cdot 5$ & 0.91 & 451 & $91 \cdot 1$ & 1.09 & $<0.01$ \\
\hline Females & 229 & 97.4 & 1.42 & 661 & 78.9 & 1.33 & $<0.01$ \\
\hline
\end{tabular}

BP, blood pressure.

${ }^{*} n$ is not the same because of missing values.

† Weighted for age and sex to the Queensland population aged over 25 for the survey year.

$\ddagger \chi^{2}$-Statistic test of association with adjustment for cluster design; $t$ test for differences in means for variables with two groups.

$\S$ Unadjusted. 
Table 2. Adjusted* geometric mean concentrations of serum carotenoids by metabolic syndrome for adults 25 years and over, who participated in the Queensland the Australian Diabetes, Obesity and Lifestyle Study 2000

(Mean values and $95 \% \mathrm{Cl} ; n$ 1523)

\begin{tabular}{|c|c|c|c|c|c|}
\hline \multirow[b]{2}{*}{ Serum carotenoid $(\mathrm{mmol} / \mathrm{l})$} & \multicolumn{2}{|c|}{$\begin{array}{l}\text { Metabolic syndrome } \\
\text { present }(n 420)\end{array}$} & \multicolumn{2}{|c|}{$\begin{array}{l}\text { Metabolic syndrome } \\
\text { absent }(n 1103)\end{array}$} & \multirow[b]{2}{*}{$P^{*}$} \\
\hline & Mean† & $95 \% \mathrm{Cl}$ & Meant & $95 \% \mathrm{Cl}$ & \\
\hline$\alpha$-Carotene & 0.10 & $0.07,0.15$ & 0.13 & $0.10,0.18$ & 0.02 \\
\hline$\beta$-Carotene & 0.45 & $0.33,0.62$ & 0.59 & $0.47,0.73$ & 0.01 \\
\hline$\beta$-Cryptoxanthin & 0.20 & $0.16,0.24$ & 0.21 & $0.18,0.25$ & 0.19 \\
\hline Lutein/zeaxanthin & 0.41 & $0.36,0.46$ & 0.41 & $0.34,0.50$ & 0.89 \\
\hline Lycopene & 0.40 & $0.34,0.47$ & 0.43 & $0.39,0.49$ & 0.31 \\
\hline Sum of serum carotenoids & 1.82 & $1.62,2.04$ & 2.02 & $1 \cdot 76,2 \cdot 33$ & 0.04 \\
\hline
\end{tabular}

* Multivariable linear regression model adjusting for potential cofounders including: age $(25-34,35-44$, $45-54,55-64,65-74$ and $75+$ ); sex (male and female); education (post-graduate qualification, trade certificate and bachelor degree, secondary school); BMI (obese, overweight and normal); alcohol intake ( $>60$ drinks per month, $<60$ drinks per month and none); smoking (current, former and never); physical activity (sedentary, insufficiently active and sufficiently active); vitamin use during $24 \mathrm{~h}$ (yes or no).

†Serum carotenoids were log transformed for regression analyses. Back-transformed geometric mean reported in table.

differences in serum carotenoid concentrations by metabolic syndrome status were found among 'current smokers'. However, among 'former' and 'never' smokers, serum $\alpha$ - and $\beta$-carotenes were significantly lower among those with metabolic syndrome than those without. In addition, among former smokers, serum lycopene was marginally lower in those with metabolic syndrome, while among never smokers, total serum carotenoids were marginally lower in those with metabolic syndrome compared with those without.

We also found no significant interaction between smoking status and serum carotenoid concentrations in relation to the metabolic syndrome status. The following are the $P$ values for interaction after adjustment for the confounding variables in relation to: $\alpha$-carotene ( $P$ for interaction $=0 \cdot 25$ ); $\beta$-carotene $(P$ for interaction $=0 \cdot 15) ; \beta$-cryptoxanthin $(P$ for interaction $=0.40)$; lutein/zeaxanthin $(P$ for interaction $=0.25)$; lycopene $(P$ for interaction $=0.41)$; total serum carotenoid $(P$ for interaction $=0 \cdot 15)$.

\section{Discussion}

The present study was designed to investigate the association between several serum carotenoids and the metabolic syndrome. The data from the present population study suggest that several serum carotenoids are inversely related to the metabolic syndrome. The present study showed significantly lower concentrations of $\alpha-\beta$-carotenes and the sum of the five carotenoids among those with the metabolic syndrome present compared with those without. We also found decreasing concentrations of all the carotenoids tested as the number

Table 3. Mean age and sex-adjusted ${ }^{*}$ concentrations of serum carotenoids by metabolic syndrome components among adults 25 years and over, who participated in the Queensland the Australian Diabetes, Obesity and Lifestyle Study 2000

(Mean values with their standard errors; $n$ 1523)

\begin{tabular}{|c|c|c|c|c|c|c|c|c|c|c|c|c|c|}
\hline & \multicolumn{12}{|c|}{ Number of metabolic syndrome components $†$ present in participants } & \multirow[b]{3}{*}{$P \ddagger$} \\
\hline & \multicolumn{2}{|c|}{$0(n 344)$} & \multicolumn{2}{|c|}{$1(n 367)$} & \multicolumn{2}{|c|}{$2(n 344)$} & \multicolumn{2}{|c|}{$3(n 250)$} & \multicolumn{2}{|c|}{$4(n 155)$} & \multicolumn{2}{|c|}{$5(n 63)$} & \\
\hline & Mean & SE & Mean & SE & Mean & SE & Mean & SE & Mean & SE & Mean & SE & \\
\hline $\begin{array}{l}\text { Proportion of participants with } \\
\text { metabolic syndrome } \\
\text { components (\%) }\end{array}$ & \multicolumn{2}{|c|}{22} & \multicolumn{2}{|c|}{24} & \multirow{2}{*}{\multicolumn{2}{|c|}{23}} & \multirow{2}{*}{\multicolumn{2}{|c|}{17}} & \multirow{2}{*}{\multicolumn{2}{|c|}{10}} & \multirow{2}{*}{\multicolumn{2}{|c|}{4}} & \\
\hline Serum carotenoid§ (mmol/l) & & & & & & & & & & & & & \\
\hline$\alpha$-Carotene & 0.21 & 0.02 & 0.18 & 0.03 & 0.17 & 0.02 & 0.15 & 0.03 & 0.12 & 0.02 & 0.10 & 0.01 & 0.003 \\
\hline$\beta$-Carotene & 0.83 & 0.09 & 0.73 & $0 \cdot 10$ & 0.68 & 0.09 & 0.60 & 0.07 & 0.48 & 0.06 & 0.48 & 0.08 & 0.004 \\
\hline$\beta$-Cryptoxanthin & 0.29 & 0.03 & 0.31 & 0.04 & 0.28 & 0.02 & 0.26 & 0.03 & 0.23 & 0.02 & 0.25 & 0.06 & 0.01 \\
\hline Lutein/zeaxanthin & 0.46 & 0.03 & 0.44 & 0.03 & 0.46 & 0.03 & 0.43 & 0.03 & 0.46 & 0.02 & 0.38 & 0.03 & 0.39 \\
\hline Lycopene & 0.62 & 0.04 & 0.60 & 0.04 & 0.56 & 0.03 & 0.49 & 0.02 & 0.48 & 0.03 & 0.38 & 0.02 & $0 \cdot 10$ \\
\hline Total serum carotenoids & 2.42 & 0.16 & $2 \cdot 27$ & 0.21 & $2 \cdot 14$ & 0.13 & 1.93 & 0.15 & 1.78 & 0.10 & 1.59 & 0.16 & 0.006 \\
\hline
\end{tabular}

* Multivariable logistic regression model adjusted for age (25-34, 35-44, 45-54, 55-64, 65-74 and 75+); sex (male and female); education (post-graduate qualification, trade certificate and bachelor degree, secondary school); BMI (obese, overweight and normal); alcohol intake ( $>60$ drinks per month, $<60$ drinks per month and none); smoking (current, former and never); physical activity (sedentary, insufficiently active and sufficiently active); vitamin use (yes or no).

† Components 0 - none of the components of metabolic syndrome are present; components 1 - any one of the metabolic syndrome components is present; components 2 - any two out of the five metabolic syndrome components are present; components 3 - any three out of the five metabolic syndrome components are present; components 4 - any four out of the five metabolic syndrome components are present; components 5 - all five metabolic syndrome components are present.

$\ddagger$ ANOVA used to test for differences in means for variables with more than two groups.

$\S$ Serum carotenoids not log transformed. 
Table 4. Risk for metabolic syndrome and its components according to quartiles of plasma serum carotenoids (OR and $95 \% \mathrm{Cl}$ values)

\begin{tabular}{|c|c|c|c|c|c|c|c|c|c|c|c|c|}
\hline \multirow[b]{2}{*}{ Serum carotenoids } & \multicolumn{2}{|c|}{$\begin{array}{l}\text { Metabolic syndrome } \\
\qquad(n \text { 424) }\end{array}$} & \multicolumn{2}{|c|}{$\begin{array}{l}\text { Abdominal obesity } \\
\qquad(n 858)\end{array}$} & \multicolumn{2}{|c|}{ High TAG $(n 474)$} & \multicolumn{2}{|c|}{$\begin{array}{l}\text { Low HDL cholesterol } \\
\text { (n 4690) }\end{array}$} & \multicolumn{2}{|c|}{$\begin{array}{l}\text { High fasting blood } \\
\text { glucose ( } n \text { 297) }\end{array}$} & \multicolumn{2}{|c|}{$\begin{array}{l}\text { High-blood pressure } \\
\qquad(n 6670)\end{array}$} \\
\hline & OR & $95 \% \mathrm{Cl}$ & OR & $95 \% \mathrm{Cl}$ & OR & $95 \% \mathrm{Cl}$ & OR & $95 \% \mathrm{Cl}$ & OR & $95 \% \mathrm{Cl}$ & OR & $95 \% \mathrm{Cl}$ \\
\hline \multicolumn{13}{|l|}{$\alpha$-Carotene $(\mu \mathrm{g} / \mathrm{d})$} \\
\hline Q1 & 1.00 & Ref & 1.00 & Ref & 1.00 & Ref & 1.00 & Ref & 1.00 & Ref & 1.00 & Ref \\
\hline Q2 & 0.76 & $0.53,1.08$ & 0.74 & $0.37,1.48$ & 0.83 & $0.59,1.18$ & 0.65 & $0.37,1.13$ & 0.89 & $0.65,1.21$ & 0.81 & $0.57,1.17$ \\
\hline Q3 & 0.59 & $0.40,0.89$ & 0.69 & $0.55,0.87$ & 0.53 & $0.36,0.78$ & 0.78 & $0.50,1.19$ & 0.72 & $0.47,1 \cdot 10$ & 0.56 & $0.35,0.91$ \\
\hline Q4 & 0.36 & $0.21,0.63$ & 0.47 & $0.33,0.65$ & 0.55 & $0.36,0.83$ & 0.46 & $0.24,0.92$ & 0.54 & $0.33,0.90$ & 0.50 & $0.42,0.59$ \\
\hline$P$ for trend & 0.005 & & $<0.01$ & & 0.011 & & 0.06 & & 0.011 & & $<0.01$ & \\
\hline \multicolumn{13}{|l|}{$\beta$-Carotene } \\
\hline Q1 & 1.00 & Ref & 1.00 & Ref & 1.00 & Ref & 1.00 & Ref & 1.00 & Ref & 1.00 & Ref \\
\hline Q2 & 0.63 & $0.51,0.77$ & 0.87 & $0.33,2.27$ & 0.70 & $0.37,1.33$ & 0.64 & $0.38,1.06$ & 0.94 & $0.47,1.90$ & 0.47 & $0.27,0.80$ \\
\hline Q3 & 0.56 & $0.35,0.89$ & 0.58 & $0.24,1.36$ & 0.46 & $0.31,0.69$ & 0.60 & $0.31,1.40$ & 0.91 & $0.71,1 \cdot 17$ & 0.48 & $0.31,0.73$ \\
\hline Q4 & 0.36 & $0.24,0.55$ & 0.57 & $0.31,1.06$ & 0.37 & $0.21,0.64$ & 0.39 & $0.20,0.76$ & 0.72 & $0.45,1.17$ & 0.35 & $0.23,0.52$ \\
\hline$P$ for trend & 0.003 & & 0.03 & & 0.004 & & 0.03 & & 0.11 & & 0.002 & \\
\hline \multicolumn{13}{|l|}{$\beta$-Cryptoxanthin } \\
\hline Q1 & 1.00 & Ref & 1.00 & Ref & 1.00 & Ref & 1.00 & Ref & 1.00 & Ref & 1.00 & Ref \\
\hline Q2 & 1.00 & $0.62,1.63$ & 0.77 & $0.43,1.37$ & 1.07 & $0.61,1.89$ & 0.97 & $0.49,1.91$ & 1.07 & $0.75,1.52$ & 0.93 & $0.41,2.09$ \\
\hline Q3 & 0.68 & $0.35,1.32$ & 0.82 & $0.29,2.29$ & 0.59 & $0.46,0.77$ & 0.58 & $0.37,0.90$ & 1.09 & $0.33,3.59$ & 0.80 & $0.44,1.46$ \\
\hline Q4 & 0.78 & $0.51,1.20$ & 0.88 & $0.47,1.64$ & 0.71 & $0.46,1.10$ & 0.59 & $0.35,1.01$ & 0.69 & $0.40,1.20$ & 0.91 & $0.43,1.94$ \\
\hline$P$ for trend & 0.07 & & 0.74 & & 0.03 & & 0.05 & & 0.41 & & 0.69 & \\
\hline \multicolumn{13}{|l|}{ Lutein/zeaxanthin } \\
\hline Q1 & 1.00 & Ref & 1.00 & Ref & 1.00 & Ref & 1.00 & Ref & 1.00 & Ref & 1.00 & Ref \\
\hline Q2 & $1 \cdot 13$ & $0.55,2.33$ & 0.91 & $0.67,1.25$ & 1.00 & $0.53,1.90$ & 0.90 & $0.45,1.80$ & 0.95 & $0.75,1.20$ & 0.97 & $0.61,1.55$ \\
\hline Q3 & $1 \cdot 12$ & $0.64,1.95$ & $1 \cdot 17$ & $0.72,1.91$ & 1.37 & $0 \cdot 85,2 \cdot 22$ & 0.55 & $0.26,1.18$ & 1.09 & $0.64,1.88$ & 0.88 & $0.55,1.41$ \\
\hline Q4 & 0.93 & $0.56,1.52$ & 0.77 & $0.36,1.67$ & $1 \cdot 19$ & $0.57,2.48$ & 0.47 & $0.21,1.05$ & 0.57 & $0.33,1.00$ & 0.95 & $0.74,1.23$ \\
\hline$P$ for trend & 0.79 & & 0.72 & & 0.37 & & 0.04 & & 0.13 & & 0.53 & \\
\hline \multicolumn{13}{|l|}{ Lycopene } \\
\hline Q1 & 1.00 & Ref & 1.00 & Ref & 1.00 & Ref & 1.00 & Ref & 1.00 & Ref & 1.00 & Ref \\
\hline Q2 & 0.98 & $0.54,1.78$ & 1.00 & $0.73,1.38$ & 1.01 & $0.47,2 \cdot 19$ & 0.93 & $0.59,1.44$ & 0.76 & $0.47,1.24$ & $1 \cdot 32$ & $0.96,1.80$ \\
\hline Q3 & 0.72 & $0.44,1 \cdot 17$ & 0.97 & $0.63,1.48$ & 0.76 & $0.48,1 \cdot 22$ & 0.64 & $0.37,1.09$ & 0.88 & $0.54,1.42$ & 1.09 & $0.80,1.50$ \\
\hline Q4 & 0.76 & $0.48,1 \cdot 21$ & 0.83 & $0.53,1.29$ & 0.75 & $0.47,1.19$ & 0.64 & $0.37,1.11$ & $1 \cdot 13$ & $0.86,1.48$ & 0.98 & $0.62,1.57$ \\
\hline$P$ for trend & 0.14 & & 0.29 & & 0.07 & & 0.06 & & 0.20 & & 0.54 & \\
\hline \multicolumn{13}{|c|}{ Total serum carotenoids } \\
\hline Q1 & 1.00 & Ref & 1.00 & Ref & 1.00 & Ref & 1.00 & Ref & 1.00 & Ref & 1.00 & Ref \\
\hline Q2 & 0.63 & $0.47,0.85$ & 0.66 & $0.45,0.98$ & 1.03 & $0.66,1.61$ & 0.63 & $0.33,1 \cdot 18$ & 0.61 & $0.26,1.43$ & 0.62 & $0.35,1 \cdot 12$ \\
\hline Q3 & 0.87 & $0.51,1.50$ & 0.86 & $0.66,1 \cdot 12$ & 0.88 & $0.68,1 \cdot 12$ & 0.68 & $0.32,1.41$ & 0.98 & $0.73,1.32$ & 0.57 & $0.34,0.94$ \\
\hline Q4 & 0.43 & $0.23,0.79$ & 0.60 & $0.35,1.02$ & 0.57 & $0.34,0.96$ & 0.41 & $0.20,1.85$ & 0.56 & $0.26,1.22$ & 0.47 & $0.27,0.81$ \\
\hline$P$ for trend & 0.04 & & $0 \cdot 10$ & & 0.03 & & 0.05 & & 0.14 & & 0.008 & \\
\hline
\end{tabular}

Q, quartile; Ref, reference.

Logistic regression adjusted for age (25-34, 35-44, 45-54, 55-64, 65-74 and 75+); sex (male and female); education (post-graduate qualification, trade certificate and bachelor degree, secondary school); alcohol intake (>60 drinks per month, $<60$ drinks per month and none); smoking (current, former and never); physical activity (sedentary, insufficiently active and sufficiently active); vitamin use (yes or no) and BMI (obese, overweight and normal). 
of the metabolic syndrome components increased. This was significant for $\alpha$-, $\beta$-carotenes, $\beta$-cryptoxanthin and total carotenoids. These findings are consistent with data reported by Ford et $a l .{ }^{(23)}$ from the NHANES III. In the NHANES III study, significantly lower concentrations of all the carotenoids, except lycopene, were found among persons with the metabolic syndrome compared with those without, after adjusting for confounding factors similar to those in the present study. The NHANES III study ${ }^{(19)}$ also found decreasing concentrations of all the carotenoids (except lycopene) and total carotenoids with increasing number of metabolic syndrome components. The present study used the criteria for metabolic syndrome as suggested by the International Diabetes Federation ${ }^{(30)}$, which requires central obesity as the major component. In the NHANES III study, central obesity is a component of the syndrome, but it is not a required component. Also we adjusted for BMI in all of our analyses, whereas the NHANES study did not. This adjustment for BMI may have attenuated the strength of the association of several carotenoids in the present study when compared with NHANES III.

We did find that all the serum carotenoids decreased as BMI status increased and this was significant for all of the carotenoids. This is consistent with findings of a prospective study by Andersen et al. ${ }^{(31)}$, in which a high BMI status was strongly associated with lower concentrations of total carotenoids.

Since serum carotenoids are considered as reliable markers of vegetable and fruit intakes, low-serum concentrations could conceivably be due to lower intakes. Our previous study ${ }^{(32)}$ did find statistically significant associations between vegetable and fruit intakes and serum levels of $\alpha$-, $\beta$ carotenes, $\beta$-cryptoxanthin and lutein-zeaxanthin. We also found fewer individuals with the highest vegetable intake with the metabolic syndrome compare with those with the lowest intakes of vegetables. However, we found no association with intake of fruit and the syndrome.

Several studies have investigated fruit and vegetable intakes in relation to the metabolic syndrome with mixed results. The NHANES study ${ }^{(19)}$ reported significantly lower intakes of fruit and vegetables among those with the syndrome present compared with those without the syndrome. To estimate the number of monthly servings of fruit and vegetables, they summed twenty-one items on the NHANES FFQ. Esmaillza$\operatorname{deh}^{(18)}$ reported a significant inverse association between fruit and vegetable intakes and the metabolic syndrome among Tehrani female teachers. Fruit and vegetable intakes were estimated from a semi-quantitative FFQ. Several studies $^{(17,33)}$ have suggested that a 'healthy' dietary pattern or one of lower nutritional risk is associated with lower risk of the metabolic syndrome. Another study ${ }^{(21)}$ of dietary patterns, however, showed no association with a 'prudent' dietary pattern, typified by a higher intake of vegetables, fruit, fish and poultry and the metabolic syndrome, but did show a positive association with a 'western' pattern characterised by high intakes of refined grains, processed meat, fried foods and red meat.

Although the assessment of fruit and vegetable intakes in the present study was relatively crude (i.e. how many serves of vegetables do you usually eat in a day?) and therefore may not accurately reflect true fruit and vegetable intakes, 
our findings of vegetable intake do appear to be consistent with those of other studies suggesting a positive relationship between vegetable intake and the risk of the metabolic syndrome. Also the present study was conducted from October to December in Queensland, Australia. These months are spring and early summer and as most of Queensland is tropical and subtropical, the availability of fruit and vegetables is abundant year round. Thus, the time of year in which the study was conducted would have had little effect on results.

Smokers have been found to have significantly lower serum carotenoid concentrations compared with non-smokers ${ }^{(34)}$. Overall, we found significantly lower concentrations of total carotenoids and the individual carotenoids, except lycopene, in smokers compared with non-smokers (data not shown). However, in relation to metabolic syndrome status, we found no significant difference in serum carotenoid levels by metabolic syndrome status among current smokers. But among former and never smokers, serum $\alpha$ - and $\beta$-carotenes were relatively lower among those with metabolic syndrome than those without. Serum lycopene was marginally lower for those with metabolic syndrome compared to those without among former smokers, while total serum carotenoids was marginally lower for those with metabolic syndrome compared to those without among never smokers. We also found no significant interaction between smoking status and serum carotenoids in relation to the metabolic syndrome.

Our findings were partly similar to that of Sugiura et al. ${ }^{(24)}$, who found inverse associations between serum $\beta$-carotene among non-smoking adults. Our findings were also similar to those of Hozawa and co-investigators ${ }^{(35)}$, who found an inverse association of total carotenoids with incidence of diabetes and several markers of glucose tolerance only among non-smokers, but no association among current smokers.

There were several limitations in the present study. We did not have accurate data on the intake of any of these carotenoids from supplements. Approximately, one-third of our participants reported taking a vitamin, mineral or herbal supplement in the $24 \mathrm{~h}$ before the examination. Although we collected information about the supplements, there was no database available that could estimate the content of these carotenoids in the supplement. From investigation of a variety of supplements that were on the market at the time, few of them contained any of these carotenoids. Approximately, $57 \%$ of women in the study were post-menopausal, and, as might be expected, had higher percentage of women with the syndrome. However, we did not account for menopause status in our analyses.

The cross-sectional design of the present study does not permit us to draw inferences regarding causality. It is not possible to conclude whether low-serum concentrations of carotenoids found in participants with the metabolic syndrome in the present study are the result of increased utilisation of these antioxidants due to the oxidative stress effects of the disease, or whether the lower concentrations are the result of lower intakes of dietary carotenoids and play a role in the pathogenesis of the syndrome.

Oxidative stress is an imbalance, which produces free radicals that overwhelm the body's antioxidant defences, and high levels of oxidative stress are known to deplete the body's reserves of antioxidants. Oxidative stress has been implicated in the pathogenesis of several components of the metabolic syndrome including glucose or insulin abnormalities $^{(4)}$, hypertension ${ }^{(36)}$ and obesity ${ }^{(37)}$. Recent studies ${ }^{(38)}$ have reported elevated markers of oxidative stress among subjects with metabolic syndrome compared with those without the syndrome. Thus, the oxidative stress nature of the metabolic syndrome may cause greater utilisation of antioxidants resulting in lower concentrations of antioxidants such as carotenoids.

Another hypothesis is that lower intakes of antioxidant-rich fruit and vegetables result in lower serum concentrations, and thus are involved in the pathogenesis of these conditions. Epidemiologic studies have found lower antioxidant intakes with components of the metabolic syndrome such as diabetes status ${ }^{(39)}$. Suzuki et al. ${ }^{(7)}$ found a significantly lower OR for high HbA1c among those with the highest intakes of carrots and pumpkin compared with a low intake group. The large European Prospective Investigation into Cancer and Nutrition-Norfolk study found that persons with higher intakes of vegetables and fruit have higher serum carotenoid concentrations and lower risk of type 2 diabetes $^{(40)}$.

While numerous observational studies have shown an inverse relationship between antioxidant intakes and serum concentrations and the risk of several of these components, clinical trials providing single or combination antioxidants as supplements have not resulted in any beneficial reduction in these conditions ${ }^{(22)}$. Further research, particularly longitudinal studies, may shed light on whether low levels of carotenoids are causally involved in the development of the syndrome, or if they are a result of these pathologies. Clinical trials based on diets high in carotenoid-rich fruit and vegetables may also provide important insights not only in relation to the prevention of components of the metabolic syndrome, but also in reducing the risk of developing the syndrome itself.

Obesity and the other components of the metabolic syndrome are increasing in most countries of the world today and will continue to increase. As populations age, and as overweight and obesity continue to escalate, especially among children, these conditions will become an increasing burden on the health system. Lifestyle interventions have been able to show dramatic reductions in the risk of diabetes among those with impaired glucose tolerance ${ }^{(41,42)}$. However, strategies for both primary and secondary prevention will be necessary to reduce the burden of obesity, diabetes and the metabolic syndrome in future years, in future generations in both developed and developing countries.

\section{Acknowledgements}

The present study was supported by the Australian Commonwealth Department of Health and Ageing, Queensland Health, Diabetes Australia, the Australian Kidney Foundation and pharmaceutical companies: Abbott Australasia Pty Ltd, Alphapharm Pty Ltd, Aventis Pharmaceutical, AstraZeneca, Aventis Pharmaceutical, Bristol-Myers Squibb Pharmaceuticals, Eli Lilly (Aust) Pty Ltd, GlaxoSmithKline, Janssen-Cilag (Aust) Pty Ltd, Merck Lipha s.a., Merck Sharp and Dohme (Aust), Novartis Pharmaceutical (Aust) Pty Ltd., Novo Nordisk Pharmaceutical Pty Ltd, Pharmacia and Upjohn Pty Ltd, 
Pfizer Pty Ltd, Roche Diagnostics, Sanofi Synthelabo (Aust) Pty Ltd, Servier Laboratories (Aust) Pty Ltd. All authors approved the final version of the present paper. T. C. was responsible for the concept and conduct of the study and preparing the manuscript. T. I. I. performed the statistical analyses and writing the results section. P. D. B. provided technical assistance on the data analyses and on writing and interpretation. C. S. Mc. C and J. E. S. provided details regarding the study methods and interpretation of findings. None of the authors had any personal or financial interest in the companies that supported the study. No conflict of interest exists.

\section{References}

1. Wilson W, D'Agostino R, Parise H, et al. (2005) Metabolic syndrome as a precursor of cardiovascular disease and type 2 diabetes mellitus. Circulation 112, 3066-3072.

2. Dekker J, Girman C, Rhodes T, et al. (2005) Metabolic syndrome and 10-year cardiovascular disease risk in the Hoorn Study. Circulation 112, 666-673.

3. Ford E, Giles W \& Dietz W (2002) Prevalence of the metabolic syndrome among US adults: findings from the Third National Health and Nutrition Examination Survey. JAMA 287, 356-359.

4. Ruhe RC \& McDonald RB (2001) Use of antioxidant nutrients in the prevention and treatment of type 2 diabetes. $\mathrm{J} \mathrm{Am} \mathrm{Coll}$ Nutr 20, 363S-369S, discussion $81 \mathrm{~S}-83 \mathrm{~S}$.

5. Ford ES, Will JC, Bowman BA, et al. (1999) Diabetes mellitus and serum carotenoids: findings from the Third National Health and Nutrition Examination Survey. Am J Epidemiol 149, $168-176$.

6. Coyne T, Ibiebele T, Baade P, et al. (2005) Diabetes mellitus and serum carotenoids: findings of a population-based study in Queensland, Australia. Am J Clin Nutr 82, 685-693.

7. Suzuki K, Ito Y, Nakamura S, et al. (2002) Relationship between serum carotenoids and hyperglycemia: a populationbased cross-sectional study. J Epidemiol 12, 357-366.

8. Hozawa A, Jacobs DR, Steffes MW, et al. (2006) Relationships of circulating carotenoid concentrations with several markers of inflammation, oxidative stress, and endothelial dysfunction: the Coronary Artery Risk Development in Young Adults (CARDIA)/Young Adult Longitudinal Trends in Antioxidants (YALTA) Study. Clin Chem 53, 447-455.

9. D’Odorico A, Martines D, Kiechl S, et al. (2000) High plasma levels of alpha- and beta-carotene are associated with a lower risk of atherosclerosis. Results from the Bruneck Study. Atherosclerosis 153, 231-239.

10. Ito Y, Suzuki K, Ishii J, et al. (2006) A population-based follow-up study on mortality from cancer or cardiovascular disease and serum carotenoids, retinol and tocopherols in Japanese inhabitants. Asian Pac J Cancer Prev 7, 533-546.

11. Daviglus ML, Orencia AJ, Dyer AR, et al. (1997) Dietary vitamin C, beta-carotene and 30-year risk of stroke: results from the Western Electric Study. Neuroepidemiology 16, 69-77.

12. Hozawa A, Jacobs DR Jr, Steffes MW, et al. (2009) Circulating carotenoid concentrations and incident hypertension: the Coronary Artery Risk Development in Young Adults (CARDIA) study. J Hypertens 27, 237-242.

13. Giovannucci E, Rimm EB, Liu Y, et al. (2002) A prospective study of tomato products, lycopene, and prostate cancer risk. J Natl Cancer Inst 94, 391-398.

14. D'Odorico A, Bortolan S, Cardin R, et al. (2001) Reduced plasma antioxidant concentrations and increased oxidative DNA damage in inflammatory bowel disease. Scand J Gastroenterol 36, 1289-1294.

15. Brazionis L, Rowley K, Itsiopoulos C, et al. (2008) Plasma carotenoids and diabetic retinopathy. Br J Nutr 13, 1-8.

16. Eitenmiller RR \& Landen WO (editors) (1999) Vitamin Analysis for the Health and Food Sciences. Boca Raton, FL: CRC Press.

17. Esmaillzadeh A, Kimiagar M, Mehrabi Y, et al. (2007) Dietary patterns, insulin resistance, and prevalence of the metabolic syndrome in women. Am J Clin Nutr 85, 910-918.

18. Esmaillzadeh A, Kimiagar M, Mehrabi Y, et al. (2006) Fruit and vegetable intakes, C-reactive protein, and the metabolic syndrome. Am J Clin Nutr 84, 1489-1497.

19. Ford ES, Mokdad AH, Giles WH, et al. (2003) The metabolic syndrome and antioxidant concentrations: findings from the Third National Health and Nutrition Examination Survey. Diabetes 52, 2346-2352.

20. Yoo S, Nicklas T, Baranowski T, et al. (2004) Comparison of dietary intakes associated with metabolic syndrome risk factors in young adults: the Bogalusa Heart Study. Am J Clin Nutr 80, 841-848.

21. Lutsey PL, Steffen LM \& Stevens J (2008) Dietary intake and the development of the metabolic syndrome: the Atherosclerosis Risk in Communities Study. Circulation 117, 754-761.

22. Liu S, Ajani U, Chae C, et al. (1999) Long-term beta-carotene supplementation and risk of type 2 diabetes mellitus: a randomised controlled trial. JAMA 282, 1073-1075.

23. Ford E, Mokdad A, Giles W, et al. (2003) The metabolic syndrome and antioxidant concentrations: findings from the Third National Health and Nutrition Examination Survey. Diabetes Care 2, 2346-2352.

24. Sugiura M, Nakamura M, Ogawa K, et al. (2008) Associations of serum carotenoid concentrations with the metabolic syndrome: interaction with smoking. Br J Nutr 29, 1-10.

25. Dunstan DW, Zimmet PZ, Welborn TA, et al. (2002) The rising prevalence of diabetes and impaired glucose tolerance: the Australian Diabetes, Obesity and Lifestyle Study. Diabetes Care 25, 829-834.

26. Dunstan DW, Zimmet PZ, Welborn TA, et al. (2002) The Australian Diabetes, Obesity and Lifestyle Study (AusDiab) methods and response rates. Diabetes Res Clin Pract 57, 119-129.

27. Friedewald WT, Levy R \& Fredrickson DS (1972) Estimation of the concentration of low-density lipoprotein cholesterol in plasma, without the use of the preparative ultracentrifuge. Clin Chem 18, 499-502.

28. Talwar D, Ha TK, Cooney J, et al. (1998) A routine method for the simultaneous measurement of retinol, alpha-tocopherol and five carotenoids in human plasma by reverse phase HPLC. Clin Chim Acta 270, 85-100.

29. Alberti K, Zimmet PZ \& Shaw JE (2006) Metabolic syndrome - a new world-wide definition. A concensus statement from the International Diabetes Federation. Diabet Med 23, $469-480$.

30. Stata Corporation (2003) Stata/SE Statistical Software: Release 8.0 for Windows. College Station, TX: Stata Corporation.

31. Andersen LF, Jacobs DR Jr, Gross MD, et al. (2006) Longitudinal associations between body mass index and serum carotenoids: the CARDIA Study. Br J Nutr 95, 358-365.

32. Coyne T, Ibiebele TI, McNaughton S, et al. (2005) Evaluation of brief dietary questions to estimate vegetable and fruit consumption - using serum carotenoids and red-cell folate. Public Health Nutr 8, 298-308.

33. Millen BE, Pencina MJ, Kimokoti RW, et al. (2006) Nutritional risk and the metabolic syndrome in women: opportunities for preventive intervention from the Framingham Nutrition Study. Am J Clin Nutr 84, 434-441. 
34. Dietrich M, Block G, Norkus EP, et al. (2003) Smoking and exposure to environmental tobacco smoke decrease some plasma antioxidants and increase - tocopherol in vivo after adjustment for dietary antioxidant intakes. Am J Clin Nutr 77, $160-166$.

35. Hozawa A, Jacobs DR Jr, Steffes MW, et al. (2006) Associations of serum carotenoid concentrations with the development of diabetes and with insulin concentration: interaction with smoking: the Coronary Artery Risk Development in Young Adults (CARDIA) Study. Am $J$ Epidemiol 163, 929-937.

36. Redon J, Oliva M, Tormos C, et al. (2003) Antioxidant activities and oxidative stress byproducts in human hypertension. Hypertension 41, 1096-1101.

37. Furukawa S, Fujita T, Shimabukuro M, et al. (2004) Increased oxidative stress in obesity and its impact on metabolic syndrome. J Clin Invest 114, 1752-1761.
38. Hansel B, Giral P, Nobecourt E, et al. (2004) Metabolic syndrome is associated with elevated oxidative stress and dysfunctional dense high-density lipoprotein particles displaying impaired antioxidative activity. J Clin Endocrinol Metab 89, 4963-4971.

39. Montonen J, Knekt P, Jarvinen R, et al. (2004) Dietary antioxidant intake and risk of type 2 diabetes. Diabetes Care 27, 362-366.

40. Sargent L, Khaw K, Bingham S, et al. (2001) Fruit and vegetable intake and population glycosylated haemoglobin levels: the EPIC-Norfolk Study. Eur J Clin Nutr 55, 342-348.

41. Tuomilehto J, Lindstrom J, Eriksson J, et al. (2001) Prevention of type 2 diabetes mellitus by changes in lifestyle among subjects with impaired glucose tolerance. New Engl J Med 344, $1343-1350$.

42. Diabetes Prevention Program Research Group (2002) Reduction in the incidence of type 2 diabetes with lifestyle intervention or metformin. New Engl J Med 346, 393-403. 